\title{
Diversidad morfológica del frijol ayocote del Carso Huasteco de México
}

\section{Morphologic diversity of ayocote beans from Huasteco Karst of Mexico}

\author{
Patricia Vargas-Vázquez ${ }^{1}$, José S. Muruaga-Martínez', Sandra E. Martínez-Villarreal ${ }^{1}$, Régulo Ruiz-Salazar², \\ Sanjuana Hernández-Delgado ${ }^{2}$ y Netzahualcóyotl Mayek-Pérez ${ }^{2 *}$ \\ ${ }^{1}$ Programa de Recursos Genéticos de Frijol. Campo Experimental Valle de México. INIFAP. Km. 13.5 carretera Los Reyes-Texcoco, 56250, Coatlin- \\ chán, Texcoco, Estado de México. México. \\ ${ }^{2}$ Centro de Biotecnología Genómica-Instituto Politécnico Nacional. Blvd. del Maestro, esquina Elías Piña s/n, Col. Narciso Mendoza Reynosa 88710 \\ Tamaulipas, México. \\ *Correspondencia: nmayek@ipn.mx
}

\begin{abstract}
Resumen. El frijol ayocote (Phaseolus coccineus L.) se cultiva en zonas templadas y subtropicales de México. La colección nacional de la especie incluye 800 accesiones y está resguardada por un banco de germoplasma nacional ubicado en Chapingo, México. En este trabajo se caracterizaron 98 accesiones de frijol ayocote de la subprovincia fisiográfica Carso Huasteco de Puebla, México con base en características fenológicas y del color y dimensión de la semilla. Los colores que predominaron fueron el negro (N1) y variantes de violeta (5R) y de beige (10YR y 7.5YR). Además, se registraron las variables climáticas de los sitios de recolección originales de cada accesión. El germoplasma se separó en 2 grupos: uno incluyó 42 accesiones con ciclo biológico tardío (días a madurez fisiológica $>110$ ), semillas grandes (peso de 100 semillas $>40 \mathrm{~g}$ ) y vainas largas (longitud $>8 \mathrm{~cm}$ ) con 1 a 3 semillas, desarrollados por selección empírica de los agricultores locales en sitios con temperaturas mínimas de 2 a $5{ }^{\circ} \mathrm{C}$, y otro incluyó 56 accesiones con ciclo precoz ( $\leq 110$ días), semilla chica (peso $\leq 40 \mathrm{~g}$ ) y vainas cortas (longitud $\leq 8 \mathrm{~cm}$ ) con 2 a 4 semillas, cultivadas en sitios con temperaturas mínimas de $0 \mathrm{a}-2{ }^{\circ} \mathrm{C}$. El cultivo del frijol ayocote del Carso Huasteco se limita a una superficie relativamente pequeña; no obstante, con base en las formas y épocas de cultivo, se observó notable variación y diferenciación fenotípica en el germoplasma local. Futuros trabajos determinarán las medidas de conservación y aprovechamiento de dicho germoplasma.
\end{abstract}

Palabras clave: Phaseolus coccineus, recursos genéticos, Puebla, diversidad morfológica.

\begin{abstract}
Ayocote beans (Phaseolus coccineus L.) are cultivated in temperate and subtropical regions in Mexico. The national collection of the species includes 800 accessions and it is preserved by one national germplasm bank places in Chapingo, Mexico. In this work 98 ayocote bean accessions from sub-province Huasteco Karst of Puebla, Mexico were characterized based on phenological traits as well as seed color and dimension traits. In addition, climate conditions from each accession original site were registered. Predominant seed colors were black (N1) and variations of purple (5R) and beige (10YR and 7.5YR). Germplasm was clustered into 2 groups of accessions: one included 42 accessions with late biological cycle (days to physiological maturity $>110$ ), large seeds (weight of 100 seeds $>40$ g), and large pods (length $>8 \mathrm{~cm}$ ) with 1 to 3 seeds, which were developed by empirical selection by local farmers in locations with minimum temperatures from 2 to $5{ }^{\circ} \mathrm{C}$. The other group included 56 accessions comprising plants with early cycle $(\leq$ 110 days), small seeds (weight $\leq 40 \mathrm{~g}$ ), and short pods (length $\leq 8 \mathrm{~cm}$ ) with 2 to 4 seeds, which are commonly grown in sites with minimum temperatures from 0 to $-2{ }^{\circ} \mathrm{C}$. Despite ayocote beans grown at Huasteco karst is limited to a relatively small surface, we found significant phenotypic variation and differentiation based on forms and growing seasons. Further work should help to determine the strategies for conservation and use of this germplasm.
\end{abstract}

Key words: Phaseolus coccineus, genetic resources, Puebla, morphologic diversity.

\section{Introducción}

Aunque México es el centro de origen del frijol ayocote o patol (Phaseolus coccineus L.), la atención que se le da ha sido insuficiente para impulsarlo como alternativa de producción. En la actualidad, la especie representa

Recibido: 26 marzo 2010; aceptado: 12 enero 2011 una magnífica alternativa de producción de alimentos en áreas marginales en el altiplano y valles altos de México (Vargas-Vázquez et al., 2007). Las actuales generaciones hemos heredado la amplia variabilidad genética y fenotípica obtenida por agricultores que domesticaron el ayocote (Miranda, 1991; Delgado-Salinas, 1988) y es nuestra responsabilidad recolectarla, conservarla y caracterizarla en un intento por facilitar su utilización y aprovechamiento en los programas de mejoramiento genético. 
Kaplan y Lynch (1999) indicaron que el cultivo de $P$. vulgaris y $P$. acutifolius probablemente se remonta a más de 2500 años en el valle de Tehuacán, Puebla, y el de $P$. vulgaris, a más de 2100 años en el valle de Oaxaca y más de 1300 años en Ocampo, Tamaulipas. Desde tiempos precolombinos, el frijol (Phaseolus spp.) constituía parte de la dieta de los antiguos pobladores junto con el maíz, calabaza y chile, entre otros cultivos (Zizumbo-Villarreal y Colunga-García-Marín, 2010). Después de la Conquista, $P$. coccineus se introdujo a Europa, donde se enriqueció y diversificó en sus formas culinarias (Debouck, 1994). Actualmente, los frijoles ayocotes se cultivan en Norte y Sur América, Europa, Asia y África. Además, los colores brillantes de sus flores rojas, blancas y rosadas han hecho que se utilice como planta ornamental en Estados Unidos de América y Europa.

En México, el frijol ayocote se cultiva como una planta anual o bianual. Hernández-Xolocotzi et al. (1979) señalaron que como planta anual se siembra en monocultivo de temporal e intercalado con maíz. Las plantas que se intercalan con maíz pueden ser de guías cortas (en climas subhúmedos con lluvias en el verano y con heladas durante el invierno), o plantas trepadoras que maduran mucho después que el maíz (en climas calientes y subhúmedos con lluvias en el verano y en áreas libres de heladas). Como planta bianual, el primer año se siembra asociado con maíz y el segundo como monocultivo o de nuevo asociado con maíz o con árboles frutales, aunque también puede encontrarse en las cercas y límites de las parcelas agrícolas, como semiperenne o perenne. En los municipios de Libres y Serdán, Puebla, el frijol ayocote se siembra en monocultivo, mientras que en regiones de los estados de Hidalgo, México y Tlaxcala se siembran solamente de 10 a 20 surcos por parcela, junto al maíz o al frijol común (P. vulgaris). La diferencia en área cultivada de ayocote entre los estados antes mencionados pudiera deberse a variaciones en la demanda de grano, las formas culinarias de preparación distintas y a que las épocas de consumo son diferentes. También se cultiva y consume $P$. coccineus en otras regiones del país, como las partes altas del estado de Chiapas, donde por lo general se asocia con maíz, y en los estados de Chihuahua, Durango y Zacatecas, el ayocote con grano blanco (patol) se consume en las fiestas y se produce junto al frijol común de temporal (Vargas-Vázquez et al., 2007).

El frijol ayocote es una especie marginada que no se considera en las estadísticas oficiales. Es probable que su superficie sembrada y producción total se incluya en las cifras del frijol común debido, en parte, a que el fitomejoramiento se ha dedicado al frijol común, la especie de mayor demanda (Acosta et al., 1991), ya que la del frijol ayocote no es generalizada.(Vargas-Vázquez et al., 2007).
Aunado a lo anterior, la variabilidad genética de los diferentes tipos y especies de Phaseolus se ha estado perdiendo por causa de diversos factores, entre los que podríamos mencionar la modernización de la agricultura, que tiende al monocultivo de variedades mejoradas que satisfagan la demanda específica del consumidor por productos uniformes; los cambios en el uso del suelo agrícola a cultivos más rentables, o bien, el no cultivo de dichas tierras por abandono o migración (Cárdenas et al., 1996; Pool et al., 1999). Basurto-Peña (2000) detectó P. coccineus cultivada, "escapada" y silvestre en Nauzontla, Xochitlán, Zacatlán y Ahuacatlán, Puebla; mientras que Ramírez-Vallejo et al. (2006) indicaron que la diversidad morfológica del frijol ayocote del estado de México se asocia estrechamente con las formas de intercambio de semillas en los mercados regionales y de utilización en los sistemas de asociación maíz-frijol.

En el territorio nacional se recolectó germoplasma criollo de frijol ayocote a partir de la década de 1950. Las muestras se depositaron principalmente en el banco de semillas del entonces Instituto Nacional de Investigaciones Agrícolas (el actual Instituto de Investigaciones Forestales, Agrícolas y Pecuarias [INIFAP] ), donde la viabilidad de la semilla se ha mantenido hasta la fecha. En la actualidad, la colección de frijol ayocote se compone de 800 accesiones y sus datos de ingreso permiten distinguirlas de acuerdo con las características físicas de los sitios de recolección original. Las accesiones se han clasificado según las subprovincias fisiográficas de origen (Vargas-Vázquez et al., 2007).

Para este trabajo se caracterizaron 98 accesiones de frijol ayocote de la subprovincia fisiográfica Carso Huasteco del estado de Puebla, México en condiciones de campo, en un sitio experimental (Chapingo, México), con base en las características fenológicas y morfológicas de la semilla con alta heredabilidad genética, con el objetivo de determinar los patrones de variabilidad fenotípica y su posible relación con el origen geográfico de recolecta de cada accesión. La hipótesis planteó que la variabilidad fenotípica del germoplasma de frijol ayocote del Carso Huasteco es amplia y se asocia con las condiciones de clima y cultivo de cada sitio de recoleccción.

\section{Materiales y métodos}

El germoplasma se recolectó en el Carso Huasteco que se ubica en la provincia Sierra Norte de Puebla, cadena montañosa que constituye el extremo sur de la sierra Madre Oriental; limita al oriente con la Llanura Costera del Golfo de México y al poniente y al sur con la Faja Volcánica Transmexicana. Se estima que la superficie aproximada del Carso Huasteco es de $4500 \mathrm{~km}^{2}$ que representan alre- 
dedor del $13 \%$ de la superficie total del estado de Puebla (INEGI, 2010). El origen geográfico del germoplasma, colector, etc. se detalla por Vargas-Vázquez et al. (2007).

El 7 de julio de 2008, en el Campo Experimental Valle de México en Chapingo, Estado de México, en condiciones de temporal, se sembraron 149 accesiones con 7 matas en un surco de $4 \mathrm{~m}$ de largo; cada mata contenía 1 a 3 plantas. De agosto a septiembre se registró la fecha de aparición de la primera flor en cada mata y de octubre a diciembre la de la primera vaina madura. La cosecha se llevó a cabo de octubre a noviembre; 51 accesiones no germinaron o no alcanzaron la madurez fisiológica debido a que se presentaron heladas tempranas; por ello, la caracterización de la semilla y los análisis se realizaron únicamente para 98 accesiones.

De acuerdo con los descriptores del International Board for Plant Genetic Resources (IBPGR, 1983), las características que se registraron en 8 repeticiones de cada accesión fueron el peso de 10 semillas (80 semillas por accesión), el patrón de moteado de la testa, las dimensiones (largo, ancho y grosor) y la forma (largo/grosor, largo/ancho, amplitud de las 2 últimas características). Con base en el cuadro de colores de Munsell (Munsell soil color chart, 2000) se determinaron las variables de color predominante, color secundario, patrón de moteado de la testa, colores del patrón de moteado y forma del hilo, color base y color secundario (claro u oscuro) de las vainas. Es importante señalar que esta carta de colores muestra a simple vista mayor poder discriminatorio entre variedades cultivadas que otras escalas de colores internacionalmente utilizadas (Griesbach y Austin, 2005).

Los descriptores registrados se diferenciaron en cuantitativos (16) y cualitativos (12). A los descriptores cuantitativos se les determinó su valor mínimo, máximo, media, desviación estándar y varianza de la población inter e intra accesiones y a los cualitativos la frecuencia y porcentaje de presencia. Las amplitudes de cada variable se analizaron con base en sus frecuencias y porcentajes de presencia. La información se sometió al análisis de componentes principales y al de conglomerados por el método de ligamiento completo, que genera clases homogéneas y un efecto de alargamiento de las jerarquías, facilitando la determinación de grupos en comparación con otros métodos, como el de ligamiento simple (Hair et al., 1992). El análisis de la información se llevó a cabo en el programa de cómputo SAS versión 6.0.3 (SAS Institute, 1989). El registro del promedio anual de temperatura mínima, máxima y precipitación de los sitios originales de colecta se obtuvo con base en información de la Comisión Nacional para el Conocimiento y Uso de la Biodiversidad (García - CONABIO, 1998, 1998a, 1998b).

\section{Resultados}

El análisis del germoplasma indicó valores similares entre accesiones y dentro de accesiones para cada parámetro medido. Las variables con mayores valores de varianza fueron los días de inicio de floración y madurez fisiológica, así como la longitud del primer pseudorracimo, tanto entre accesiones como dentro de éstas (Cuadro 1). En cuanto a las características en vainas y semillas, se observaron valores similares en los parámetros medidos entre y dentro de accesiones. En este caso, las características con mayores valores de varianza fueron el peso de 10 semillas y la longitud de la vaina (Cuadro 2). Características tales como la dimensión de los foliolos terminales y la dimensión de las semillas muestran escasa variación (Cuadros 1, 2). En el caso de las características cualitativas, se realizaron estimaciones de la frecuencia de presencia y luego se expresaron en porcentajes. El color de la semilla fue la característica más variable en el germoplasma de frijol ayocote. Se observaron 11 clases de color predominante, 7 de color secundario, 6 tipos de color oscuro secundario, y 7 tipos de patrón del moteado (Fig. 1). Los colores predominantes fueron negro (N1) y variantes de violeta (5R) y de beige (10YR), y en cuanto a los colores secundarios, los más frecuentes fueron el café (5YR y $2.5 \mathrm{YR}$ ) y el gris (N5). Menos del 30\% del germoplasma mostró moteado de la semilla; en las accesiones que sí mostraron moteado, el patrón rayado fue el más frecuente (Cuadro 3). La forma de semilla más comunmente observada $(>80 \%)$ en las accesiones fue una combinación o variante de las 3 formas de $P$. coccineus: riñón, rectangular y/o redonda, así como el hilo oval (casi $80 \%$ ). Las relaciones largo/ancho y largo/ grueso más recurrentes en el germoplasma fueron 1.5-1.69 y mayor a 2.5 , respectivamente (Cuadro 4). En las vainas no hay patrón de moteado; cuando lo hubo, generalmente fue negro (N1). En la mayoría de los casos, el color base de las vainas fue amarillo con tonalidades tendientes al beige, naranja o café (7.5 YR) (Cuadro 5).

El análisis de componentes principales (ACP) de variables de semilla explicó el $80 \%$ del total de la variación fenotípica con los primeros 3 componentes principales (CP) (Cuadro 6). Las variables grosor de semillas (CP1), largo y relación largo/ancho de semilla (CP2) y patrón de moteado, tipo de patrón de moteado y color más oscuro (CP3) fueron altamente explicativas de la variabilidad fenotípica de la semilla de las accesiones de ayocote del Carso Huasteco (Cuadro 7).

$\mathrm{El}$ análisis de conglomerados separó las accesiones en 2 grupos (A y B). El grupo A incluyó germoplasma con floración ( 50 a 60 días después de la emergencia, -dde-) y madurez fisiológica tardía (110 a 120 dde), semillas grandes $(5 \mathrm{a} 6 \mathrm{~g})$ y vainas largas $(9 \mathrm{a} 10 \mathrm{~cm})$ con 1 a 3 semillas 
Cuadro 1. Estadísticos básicos de las características de la planta de frijol ayocote del Carso Huasteco. Chapingo, México

\begin{tabular}{|c|c|c|c|c|c|}
\hline Característica & Mínimo & Máximo & Media & $\begin{array}{l}\text { Desviación } \\
\text { estándar }\end{array}$ & Varianza \\
\hline & \multicolumn{5}{|c|}{ Entre accesiones $(\mathrm{n}=112)$} \\
\hline Días a inicio de floración & 39 & 94 & 62 & 11 & 127 \\
\hline Días a inicio de madurez & 100 & 139 & 116 & 8 & 59 \\
\hline Largo del foliolo terminal (cm) & 6.1 & 9.4 & 7.6 & 0.7 & 0.4 \\
\hline Ancho del foliolo terminal & 5.1 & 7.3 & 6.2 & 0.5 & 0.2 \\
\hline \multirow[t]{2}{*}{ Longitud del primer pseudorracimo $(\mathrm{cm})$} & 24.3 & 69.3 & 40.9 & 7.5 & 56.6 \\
\hline & \multicolumn{5}{|c|}{ Dentro de accesiones $(n=489)$} \\
\hline Días a inicio de floración & 39 & 95 & 55 & 10 & 103 \\
\hline Días a inicio de madurez & 98 & 139 & 112 & 8 & 61 \\
\hline Largo del foliolo terminal (cm) & 5.5 & 10.4 & 7.6 & 0.8 & 0.7 \\
\hline Ancho del foliolo terminal & 4.7 & 8.9 & 6.2 & 0.6 & 0.4 \\
\hline Longitud del primer pseudorracimo $(\mathrm{cm})$ & 16.3 & 69.3 & 40.1 & 8.9 & 78.2 \\
\hline
\end{tabular}

Cuadro 2. Estadísticos básicos de las características de la vaina y la semilla del frijol ayocote del Carso Huasteco. Chapingo, México

\begin{tabular}{|c|c|c|c|c|c|}
\hline Característica & Mínimo & Máximo & Media & $\begin{array}{c}\text { Desviación } \\
\text { estándar }\end{array}$ & Varianza \\
\hline & \multicolumn{5}{|c|}{ Entre accesiones $(\mathrm{n}=112)$} \\
\hline Largo de vaina (cm) & 7.6 & 11.8 & 9.3 & 0.9 & 0.7 \\
\hline Ancho de vaina $(\mathrm{cm})$ & 1.1 & 2.1 & 1.4 & 0.1 & 0.02 \\
\hline Lóculos por vaina & 2 & 6 & 4 & 1 & 0.2 \\
\hline Semillas por vaina & 2 & 4 & 3 & 1 & 0.2 \\
\hline Largo de semilla (cm) & 1.2 & 1.8 & 1.5 & 0.1 & 0.02 \\
\hline Ancho de semilla (cm) & 0.8 & 1.2 & 1.0 & 0.1 & 0.01 \\
\hline Grosor de semilla (cm) & 0.5 & 0.7 & 0.6 & 0.1 & 0.01 \\
\hline Peso de 10 semillas (g) & 3.6 & 11.4 & 6.3 & 1.3 & 1.7 \\
\hline Relación largo/ancho & 1.3 & 1.8 & 1.6 & 0.1 & 0.01 \\
\hline \multirow[t]{2}{*}{ Relación largo/grosor } & 2.0 & 2.9 & 2.5 & 0.2 & 0.04 \\
\hline & \multicolumn{5}{|c|}{ Dentro de accesiones $(n=489)$} \\
\hline Largo de vaina $(\mathrm{cm})$ & 6.0 & 12.6 & 9.3 & 1.2 & 1.5 \\
\hline Ancho de vaina $(\mathrm{cm})$ & 1.0 & 2.9 & 1.4 & 0.2 & 0.03 \\
\hline Lóculos por vaina & 2 & 7 & 4 & 1 & 0.4 \\
\hline Semillas por vaina & 1 & 5 & 3 & 1 & 0.4 \\
\hline Largo de semilla (cm) & 1.0 & 2.0 & 1.5 & 0.2 & 0.03 \\
\hline Ancho de semilla (cm) & 0.7 & 1.3 & 1.0 & 0.1 & 0.01 \\
\hline Grosor de semilla $(\mathrm{cm})$ & 0.4 & 0.9 & 0.6 & 0.1 & 0.01 \\
\hline Peso de 10 semillas (g) & 2.3 & 11.8 & 6.4 & 1.8 & 3.1 \\
\hline Relación largo/ancho & 0.9 & 2.1 & 1.6 & 0.1 & 0.02 \\
\hline Relación largo/grosor & 1.6 & 3.7 & 2.5 & 0.3 & 0.1 \\
\hline
\end{tabular}

por vaina. El grupo B incluyó accesiones con floración (40 a 60 dde) y madurez fisiológica (100 a 110 dde) precoz, semillas chicas (4 a $6 \mathrm{~g}$ ) y vainas cortas $(8 \mathrm{a} 9 \mathrm{~cm}) \operatorname{con} 2 \mathrm{a}$ 4 semillas por vaina (Fig. 2).

\section{Discusión}

El germoplasma de $P$. coccineus analizado mostró variabilidad morfológica alta a pesar de su origen geográfico hasta cierto punto restringido $\left(4500 \mathrm{~km}^{2}\right.$ aproxi- madamente, y 2 tipos de clima: cálido-húmedo y templado húmedo; INEGI, 2010), aunque también se observó predominancia de algunas características morfológicas registradas. En el caso del color de la semilla predominaron las tonalidades oscuras (violeta, morado, gris, café, negro) con escasa presencia de moteado. De igual modo, con base en el ciclo biológico, el germoplasma pudo separarse claramente en precoces y tardíos. Los genotipos precoces se distinguieron por sus semillas y vainas más chicas y con mayor número de semillas, en comparación 

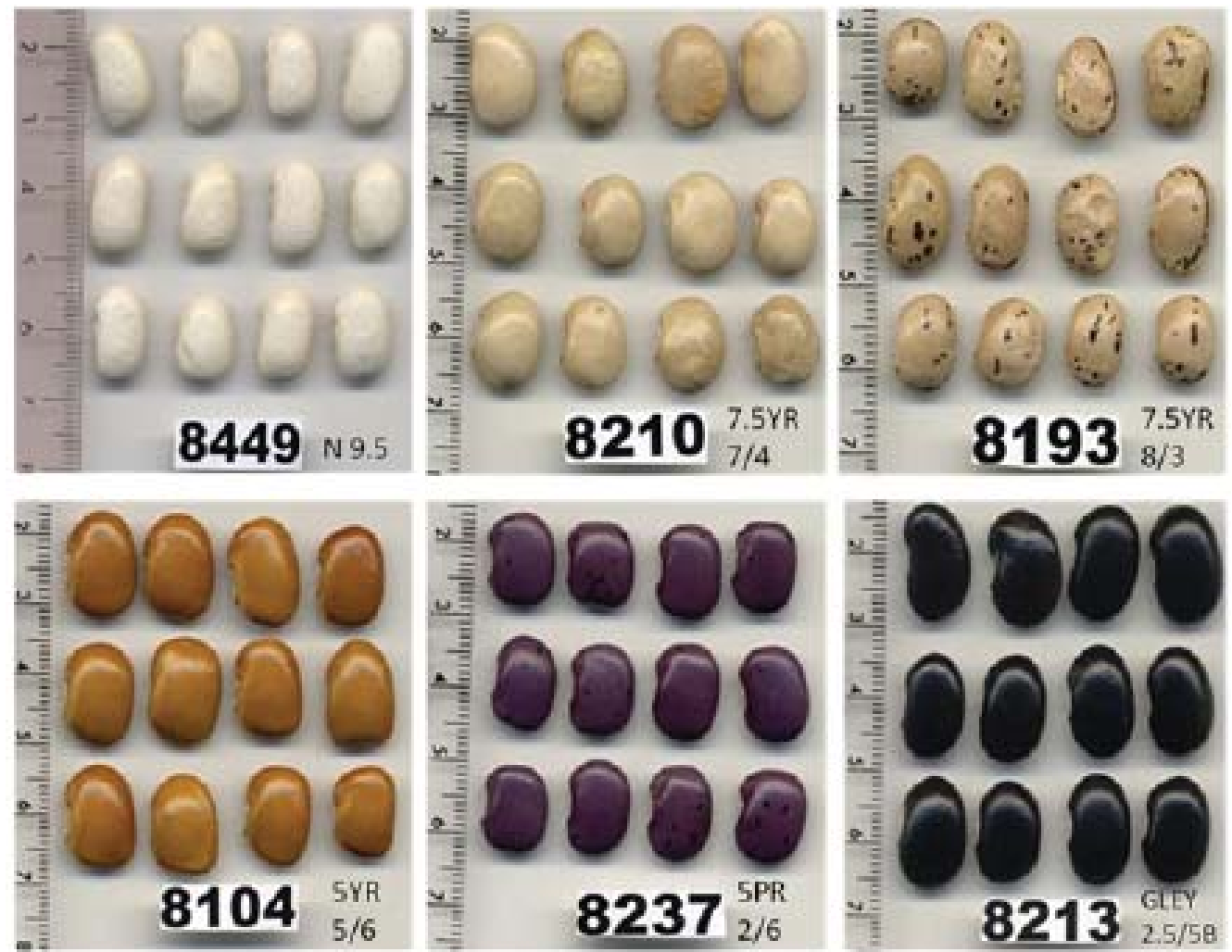

Figura 1. Algunos colores de testa de la semilla del germoplasma de frijol ayocote del Carso Huasteco, México; donde 8449, blanco (N95); 8210, beige (7.5YR 7/4); 8193, beige con manchas negras (7.5YR 8/3); 8104, café (5YR 5/6); 8237, violeta (5PR 2/6); 8213, negro (N1). Las claves entre paréntesis indican el color de acuerdo con el cuadro de colores Munsell soil color chart, 2000.

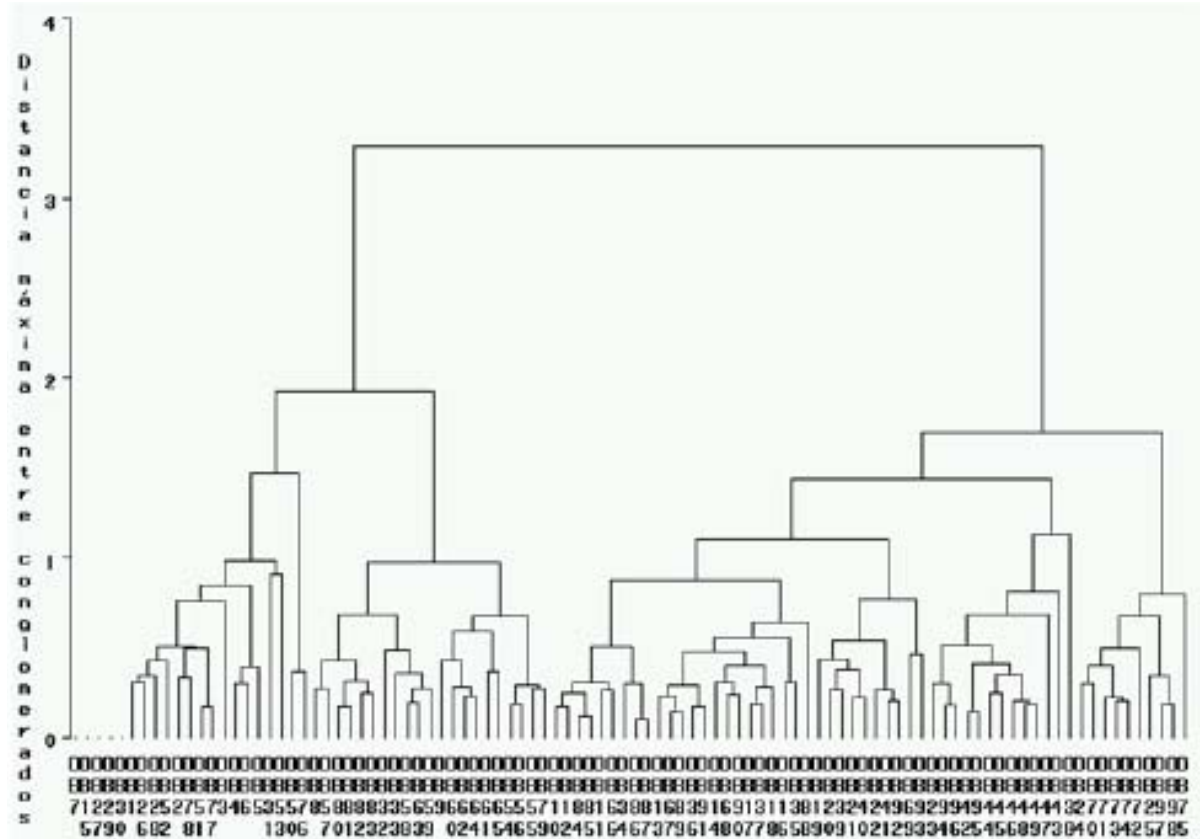

Figura 2. Dendrograma de 96 accesiones de frijol ayocote de la sub-provincia fisiográfica Carso Huasteco de Puebla, México, con base en caracteres morfológicos. 
Cuadro 3. Distribución de frecuencias porcentuales del color y patrón de moteado de la semilla del frijol ayocote del Carso Huasteco. Chapingo, México

\begin{tabular}{|c|c|c|c|c|c|c|c|c|c|}
\hline \multicolumn{6}{|c|}{ Color $^{1}$} & \multicolumn{4}{|c|}{ Moteado 2} \\
\hline Predominante & $\%$ & Claro secundario & $\%$ & $\begin{array}{c}\text { Oscuro } \\
\text { secundario }\end{array}$ & $\%$ & Patrón & $\%$ & Tipo de patrón & $\%$ \\
\hline N 9.5 & 2.9 & Ausente & 88.3 & Ausente & 80.7 & Ausente & 71.7 & Ausente & 71.9 \\
\hline $\begin{array}{c}\text { 5YR 5/6, 2.5YR } \\
5 / 4,2.5 \mathrm{YR} 3 / 4\end{array}$ & 3.1 & $\begin{array}{c}5 \mathrm{YR} 5 / 6,2.5 \mathrm{YR} \\
5 / 4\end{array}$ & 3.3 & 5YR 5/6 & 0.6 & Un color & 25.4 & Salpicado & 1.8 \\
\hline $5 \mathrm{R} 2 / 6$ & 12.5 & $5 \mathrm{R} 2 / 6$ & 0.6 & $5 \mathrm{R} 2 / 6$ & 0.2 & Dos colores & 2.9 & Rayado & 11.7 \\
\hline $5 \mathrm{R} 1 / 4$ & 11.5 & $5 \mathrm{R} 1 / 4$ & 1.2 & $5 \mathrm{R} 1 / 4$ & 0.2 & & & Intensamente & 1.6 \\
\hline N1 & 28.3 & $5 \mathrm{R} 5 / 6,4 / 6,3 / 4$ & 1.2 & N1 & 18.0 & & & $\begin{array}{c}\text { rayado } \\
\text { Casi continuo }\end{array}$ & 6.6 \\
\hline $\begin{array}{c}10 \mathrm{YR} 8 / 3,7.5 \mathrm{YR} \\
7 / 4,7.5 \mathrm{YR} 8 / 3\end{array}$ & 19.2 & $\begin{array}{c}10 \mathrm{YR} 8 / 3,7.5 \mathrm{YR} \\
7 / 4,7.5 \mathrm{YR} 8 / 3\end{array}$ & 1.4 & $5 \mathrm{~PB} 3 / 2$ & 0.2 & & & Punteado & 2.1 \\
\hline $5 \mathrm{R} 5 / 6,4 / 6,3 / 4$ & 17.6 & N5 & 3.9 & & & & & $\begin{array}{c}\text { Dos o más } \\
\text { patrones de los } \\
\text { antes citados }\end{array}$ & 4.3 \\
\hline 10 YR $6 / 8,5 / 8$ & 3.3 & & & & & & & & \\
\hline $5 \mathrm{R} 1 / 10$ & 1.2 & & & & & & & & \\
\hline 10YR 5/6 & 0.2 & & & & & & & & \\
\hline $5 \mathrm{R} 1 / 6$ & 0.2 & & & & & & & & \\
\hline
\end{tabular}

${ }^{1}$ De acuerdo con Munsell soil color chart, 2000.

${ }^{2}$ De acuerdo con IBPGR, 1983.

Cuadro 4. Distribución de frecuencias porcentuales de la forma de la semilla de frijol ayocote del Carso Huasteco. Chapingo, México

\begin{tabular}{|c|c|c|c|c|c|c|c|}
\hline \multirow[b]{2}{*}{ Semilla } & \multicolumn{3}{|c|}{ Forma $^{1}$} & \multicolumn{4}{|c|}{ Relaciones } \\
\hline & $\%$ & Hilo & $\%$ & Largo/Ancho & $\%$ & Largo/Grueso & $\%$ \\
\hline Riñón & 0.8 & Lineal & 21.3 & $0.9-1.19$ & 0.6 & $1.41-1.69$ & 0.2 \\
\hline Rectangular & 14.8 & Oval & 78.7 & $1.2-1.29$ & 0.4 & $1.7-1.80$ & 0.4 \\
\hline Mezclada o variable (riñón, rectangular y/o redonda) & 83.4 & & & $1.3-1.49$ & 22.1 & $1.81-2.09$ & 10.0 \\
\hline \multirow[t]{3}{*}{ Redonda } & 1.0 & & & $1.5-1.69$ & 58.6 & $2.1-2.2$ & 12.9 \\
\hline & & & & $1.7-1.89$ & 17.0 & $2.21-2.49$ & 34.9 \\
\hline & & & & 1.9 a más & 1.2 & 2.5 o más & 41.6 \\
\hline
\end{tabular}

${ }^{1}$ De acuerdo con IBPGR, 1983.

Cuadro 5. Distribución de frecuencias porcentuales de las características de la vaina de frijol ayocote del Carso Huasteco. Chapingo, México

\begin{tabular}{cccccc}
\hline Patrón de moteado $^{1}$ & $\%$ & Color del patrón de moteado & & \% & Color base de la vaina \\
& & & & \\
\hline Ausente & 67.9 & Ausente & 67.9 & $7.5 \mathrm{GY}$ & 0.6 \\
Presente & 32.1 & $5 \mathrm{R} 2 / 6$ & 1.7 & $7.5 \mathrm{YR}$ & 98.5 \\
& & $\mathrm{~N} 1$ & 30.4 & $5 \mathrm{YR}$ & 0.2 \\
& & & $10 \mathrm{YR}$ & 0.6 \\
\hline
\end{tabular}

${ }^{1}$ De acuerdo con IBPGR, 1983.

${ }^{2}$ De acuerdo con Munsell soil color chart, 2000.

con los genotipos tardíos, que exhibieron semillas y vainas grandes y con pocas semillas. Podría considerarse también que el llenado y menor número de vainas y de semillas en germoplasma tardío es consecuencia de la menor presencia de polinizadores (insectos, aves) durante su floración que ocurrió con temperaturas menos favorables para ello, a diferencia de su mayor incidencia durante la floración de germoplasma con floración precoz (M. L. P. VargasVázquez, INIFAP-Chapingo, comunicación personal). El germoplasma precoz crece en temperaturas más frías que el tardío. Esta diferenciación fisiológica permite inferir los efectos de la selección empírica que los agricultores de la región han realizado por años, los que se determinan tanto por las condiciones ambientales imperantes como por las 
Cuadro 6. Valores característicos del análisis de componentes principales de frijol ayocote del Carso Huasteco de Puebla, México

\begin{tabular}{cccc}
\hline $\begin{array}{c}\text { Componente } \\
\text { principal }\end{array}$ & $\begin{array}{c}\text { Valor } \\
\text { característico }\end{array}$ & $\begin{array}{c}\text { Varianza } \\
\text { explicada } \\
\text { (\%) }\end{array}$ & $\begin{array}{c}\text { Varianza } \\
\text { explicada } \\
\text { acumulada } \\
\text { (\%) }\end{array}$ \\
\hline 1 & 3.88 & 48.6 & 48.6 \\
2 & 1.54 & 19.3 & 67.9 \\
3 & 1.04 & 13.0 & 80.9 \\
4 & 0.59 & 7.3 & 88.2 \\
5 & 0.50 & 6.3 & 94.5 \\
\hline
\end{tabular}

Cuadro 7. Vectores característicos de las variables medidas en germoplasma de frijol ayocote del Carso Huasteco de Puebla, México, para los primeros 3 componentes principales del ACP

\begin{tabular}{lccc}
\hline Variable & $C P 1$ & $C P 2$ & $C P 3$ \\
\hline Peso de 10 semillas & -0.41 & 0.23 & 0.27 \\
Grosor de semilla & $\underline{-0.44}$ & 0.01 & 0.27 \\
Ancho de semilla & -0.40 & 0.10 & 0.22 \\
Largo de semilla & -0.25 & $\underline{-0.44}$ & 0.25 \\
Rango del largo/grosor de semilla & 0.23 & 0.40 & -0.04 \\
Relación largo/ancho & 0.18 & $\underline{0.44}$ & 0.06 \\
Relación largo/grosor & 0.29 & 0.38 & -0.09 \\
Rango del largo/ancho de semilla & 0.18 & 0.42 & 0.04 \\
Patrón de moteado & 0.28 & -0.16 & $\underline{0.49}$ \\
Tipo de patrón de moteado & 0.29 & -0.13 & $\underline{0.49}$ \\
Color más oscuro & 0.24 & -0.11 & $\underline{0.50}$ \\
Venación de la semilla & -0.01 & -0.15 & -0.02 \\
\hline
\end{tabular}

Los valores subrayados son los más altamente explicativos.

preferencias del agricultor mismo y por las del mercado regional, lo que es más evidente en el caso de los colores de las semillas, que indica que el consumidor local prefiere ayocotes con la testa de color oscuro. Phaseolus coccineus es una especie apreciada en la cocina de algunos países europeos, donde prefieren el grano claro, beige o blanco (Santalla et al., 2004).

En las localidades de Ahuacatlán, Ciudad Serdán, Cuetzalan, Nauzontla, Tlatlauquitepec y Zacapoaxtla tienen germoplasma precoz y tardío; en tanto que en Zacatlán y Zoquiapan sólo tardío, y en las localidades de Atempan, Chignahuapan, Teteles de Ávila Castillo y Xochiapulco sólo precoz. La mayoría de las accesiones (65.6\%) proviene de sitios con temperatura mínima promedio anual de 0 a $2{ }^{\circ} \mathrm{C}$, el $21.51 \%$ de sitios con 2 a $6{ }^{\circ} \mathrm{C}$ y $12.9 \%$ de sitios con temperaturas de -6 a $0{ }^{\circ} \mathrm{C}$. Por otra parte, $73.12 \%$ proviene de lugares con temperatura máxima de 22 a $26{ }^{\circ} \mathrm{C}$ y $26.88 \%$ de sitios con temperaturas de 26 a $30{ }^{\circ} \mathrm{C}$. En la región de colecta, la precipitación total anual varió de 600 $800 \mathrm{~mm}$ a $4000-4500 \mathrm{~mm}$ y los intervalos más comunes fueron 1 500-1 800 y 2 000-2 500 mm. El germoplasma precoz se ha desarrollado mediante selección empírica en sitios con temperatura mínima de 0 a $-2{ }^{\circ} \mathrm{C}$ y el tardío en sitios con temperatura mínima de 2 a $5{ }^{\circ} \mathrm{C}$. Miranda (1991) mencionó que la temperatura es uno de los factores ecológicos que ha limitado la dispersión de las poblaciones silvestres de $P$. coccineus. Lo anterior indica que durante el proceso de selección del frijol ayocote por los productores, el germoplasma con ciclo biológico corto mostró mayor adaptación a los sitios donde la temperatura mínima durante los meses más fríos del año es cercana a $-2{ }^{\circ} \mathrm{C}$ y con 1500 a $2500 \mathrm{~mm}$ de precipitación anual; mientras que los tardíos se desarrollan en sitios con temperaturas mayores a $2{ }^{\circ} \mathrm{C}$ y con precipitación anual de 1500 a 3500 mm. Los resultados del trabajo permiten no rechazar la hipótesis experimental, en el sentido de que existe amplia variabilidad genética en el germoplasma de frijol ayocote del Carso Huasteco y que, además, dicha variabilidad se asocia con el clima y las condiciones de cultivo de cada sitio de colecta. Los estudios botánicos y de diversidad en $P$. coccineus de México son escasos. Por ejemplo, BasurtoPeña (2000) estudió algunos aspectos etnobotánicos de $P$. coccineus L. en 5 comunidades del norte de Puebla, entre los 1400 y 1800 m snm, en los municipios de Ahuacatlán, Nauzontla, Xochitlán y Zacatlán; y en todos los sitios de estudio registró la presencia y distribución de la especie en sus formas cultivada, "escapada" del cultivo y silvestre. Por su parte, Ramírez-Vallejo et al. (2006) caracterizaron morfológicamente 42 poblaciones de frijol ayocote del estado de México. La diversidad entre accesiones la atribuyeron al intercambio de semillas en los mercados regionales y por su utilización en los sistemas de asociación maízfrijol. Este trabajo constituye un primer acercamiento al conocimiento y medición del estado actual de los recursos genéticos de $P$. coccineus en una de las regiones agroecológicas más importantes del desarrollo, adaptación y distribución del frijol ayocote. Los resultados evidencian la considerable variabilidad genética en el Carso Huasteco que es indispensable caracterizar a profundidad para determinar sus posibles usos y aprovechamiento, así como para identificar estrategias de conservación in situ y/o ex situ. A este respecto, Vargas-Vázquez et al. (2007) han dado el primer paso al diagnosticar el estado actual de la especie en nuestro país e iniciar la recolección en sus lugares de adaptación y distribución. El presente trabajo continúa con el análisis de los recursos genéticos de $P$. coccineus en Carso Huasteco, una de las regiones fisiográficas más representativas del país.

\section{Agradecimientos}

Los autores agradecen el financiamiento para este trabajo por parte del Instituto Nacional de Investigaciones 
Forestales, Agrícolas y Pecuarias, del Servicio Nacional de Inspección y Certificación de Semillas-Sistema Nacional de Recursos Fitogenéticos para la Alimentación y la Agricultura, del Instituto Politécnico Nacional y del FOMIX-Gobierno del Estado de Veracruz (proyecto VER2008-C01-94070) así como los costos de publicación cubiertos por el Fondo Mixto de Fomento a la Investigación Científica y Tecnológica CONACYT-Gobierno del Estado de Tamaulipas. Sanjuana Hernández-Delgado y Netzahualcóyotl Mayek-Pérez son becarios de los programas de Estímulos al Desempeño de los Investigadores y de la Comisión de Operación y Fomento a las Actividades Académicas del IPN, y del Sistema Nacional de Investigadores del CONACYT. Se agradecen los comentarios de tres revisores.

\section{Literatura citada}

Acosta, J. A., J. S. Muruaga y F. Cárdenas. 1991. Utilización y disponibilidad de recursos genéticos de Phaseolus en México. In Avances en el estudio de los recursos fitogenéticos de México, R. Ortega, G. Palomino, F. Castillo, V. González y M. Livera. (eds.). SOMEFI, Chapingo, Estado de México. p. 187-196.

Basurto-Peña, F. A. 2000. Aspectos etnobotánicos de Phaseolus coccineus L. y Phaseolus polyanthus Greenman en la sierra Norte de Puebla, México. Tesis maestría, Facultad de Ciencias, Universidad Nacional Autónoma de México, México, D. F. 96 p.

Cárdenas, R. F., J. S. Muruaga y J. A. Acosta. 1996. Catálogo: banco de germoplasma de Phaseolus spp. del Instituto Nacional de Investigaciones Forestales y Agropecuarias. CONABIO/ INIFAP-Centro de Invstigaciones de la Región Centro, Campo Experimental Valle de México, México, D. F. $421 \mathrm{p}$.

Debouck, D. G. 1994. Beans (Phaseolus spp.). In Neglected crops: 1492 from a different perspective, J. E. Hernando y J. León (eds.). Plant Production and Protection Series 26. FAO, Rome. p. 47-62.

Delgado-Salinas, A. 1988. Variation, taxonomy, domestication and germplasm potentialities of Phaseolus coccineus. In Genetic Resources of Phaseolus Beans, P. Gepts (ed.). Kluwer Academic, Dordrecht. p. 441-463.

Freytag, G. F. y D. G. Debouck. 2002. Taxonomy, distribution and ecology of the genus Phaseolus (LeguminosaePapilionoideae) in North America, Mexico and Central America. Sida, Botanical Miscellany 23:1-300.

García, E. - CONABIO (Comisión Nacional para el Conocimiento y Uso de la Biodiversidad). . 1998. Precipitación total anual, escala 1:000000. México, D. F.

García, E. - CONABIO (Comisión Nacional para el Conocimiento y Uso de la Biodiversidad). 1998a.. Temperatura máxima promedio. Escala 1:1000000. CONABIO, México, D. F.

García, E .- CONABIO (Comisión Nacional para el Conocimiento y Uso de la Biodiversidad). 1998b. Temperatura mínima promedio. Escala 1:1000000. CONABIO, México, D. F.

Griesbach, R. J. y S. Austin. 2005. Comparison of the Munsell and Royal Horticultural Society's color charts in describing flower color. Taxon 54:771-773.

Hair, J. F. Jr., R. E. Anderson, R. L. Tatum y W. C. Black. 1992. Multivariate data analysis. MacMillan, New York. 544 p.

Hernández-X., E., S. Miranda y C. Prywer. 1959. El origen de Phaseolus coccineus L. darwinianus Hdz. X. et Miranda C. subspecies nova. Revista de la Sociedad Mexicana de Historial Natural 20:99-121.

Hernández-X., E., A. Ramos y M. A. Martínez. 1979. Etnobotánica. In Contribuciones al conocimiento del frijol (Phaseolus) en México, E. Mark Engleman (ed.). Colegio de Postgraduados, Chapingo, Estado de México. p. 321-333.

IBPGR (International Board for Plant Genetic Resources).1983. Phaseolus coccineus descriptors. Rome. 32 p.

INEGI (Instituto Nacional de Estadística, Geografía e Informática). 2010. Fisiografía de México. Disponible en: http://mapserver.inegi.org.mx/map/datos_basicos/ fisiografia/?s=geo\&c=937. Última consulta: 27.VIII.2010.

Kaplan, L. y T. E. Lynch. 1999. Phaseolus (Fabaceae) in archaeology: AMS radiocarbon dates and their significance for pre-Colombian agriculture. Economic Botany 53:261272.

Miranda, C. S. 1990. Identificación de las especies cultivadas del género Phaseolus. Centro de Genética, Colegio de Postgraduados, Montecillo, Estado de México. 20 p.

Miranda, C. S. 1991. Evolución de P. vulgaris y P. coccineus. In Contribuciones al conocimiento del frijol (Phaseolus) en México, E. M. Engleman (ed.). Colegio de Postgraduados. Chapingo, Estado de México. p. 83-99.

Munsell soil color chart. 2000. Macbeth Division, Kollmorgen, Baltimore.

Pool, N. L., A. Trinidad, J. D. Etchevers, J. Pérez y A. Martínez. 1999. Mejoradores de la fertilidad del suelo en la agricultura de ladera de los altos de Chiapas, México. Agrociencia 34:251-259.

Ramírez-Vallejo, P., M. C. Mendoza-Castillo, F. CastilloGonzález y S. Miranda-Colín. 2006. Diversidad morfológica de poblaciones nativas de frijol común y frijol ayocote del oriente del Estado de México. Revista Fitotecnia Mexicana 29:111-119.

Santalla, M., M. C. Menéndez-Sevillano, A. B. Monteagudo y A. M. de Ron. 2004. Genetic diversity of Argentinean common bean and its evolution during domestication. Euphytica 135:75-87.

SAS Institute Inc. 1989. Statistical Analysis System versión 6.0.3. Cary, NC, USA; última consulta: 05.V.2010.

Vargas-Vázquez, M. L. P., J. S. Muruaga-Martínez, J. M., 
Hernández-Casillas y J. Díaz-De la Cruz. 2007. Diagnóstico de la forma cultivada del frijol ayocote Phaseolus coccineus L. e información geográfica de los sitios de colecta. Campo Experimental Valle de México, INIFAP, Chapingo, Estado de
México. $18 \mathrm{p}$

Zizumbo-Villarreal, D. y P. Colunga-García-Marín. 2010. Origin of agriculture and plant domestication in West Mesoamerica. Genetic Resources and Crop Evolution 6:813-825. 